\title{
IN MEMORY OF ALOIS APFELBECK: AN INTERCONNECTION BETWEEN CAYLEY-EISENSTEIN-PÓLYA AND LANDAU PROBABILITY DISTRIBUTIONS
}

\author{
VLADIMÍR VOJTA*
}

\begin{abstract}
Chotovická 12, 18200 Praha 8
\end{abstract}
* corresponding author: vojta@karneval.cz

Abstract. The interconnection between the Cayley-Eisenstein-Pólya distribution and the Landau distribution is studied, and possibly new transform pairs for the Laplace and Mellin transform and integral expressions for the Lambert $W$ function have been found.

Keywords: Cayley-Eisenstein-Pólya distribution, Landau distribution, Lambert function, Laplace transform, Mellin transform, Mellin multiplier, Hadamard fractional integral, Liouville fractional integral.

AMS Mathematics Subject Classification: 33E99, (44A10, 44A15, 26A33).

\section{INTRODUCTION}

In their seminal paper on queueing theory [1] Abate and Whitt studied a cumulative probability distribution function (c.d.f.) $F(x)$ with a pertinent probability density function (p.d.f.) $f(x), x>0$, such that its moment generating function

$$
G(s)=\int_{0}^{\infty} e^{-s x} d F(x)=\int_{0}^{\infty} e^{-s x} f(x) d x,
$$

satisfies the functional equation

$$
G(s)=e^{-s G(s)}
$$

It was shown in [1] that the moments of $F(x)$ are

$$
m_{n}=(n+1)^{n-1}, \quad n \geq 0 .
$$

The authors of [1] named this probability distribution the Cayley-Eisenstein-Pólya (C.E.P.) distribution. The solution of the functional equation (1.2) was not carried out in [1]. The primary effort of the author was to solve this problem with the aim to obtain an explicit formula for the probability density function $f(x)$. During the calculation of $f(x)$ by three methods, it was found that the C.E.P. distribution is in relation to the Landau distribution function [2].

Theorem 1.1. The solution of functional equation (1.2) is the function $G(s)=W(s) / s=e^{-W(s)}, s \in \mathbb{C}$, where $W(s)$ is the Lambert function [3] and $\mathbb{C}$ is the set of all complex numbers.

Proof. Let $U(s)=s G(s)$. Then Eq. 1.2 can be rewritten as $U(s) e^{U(s)}=s$. But this is the definition of the Lambert $W$ function as a solution of the functional equation $W(s) e^{W(s)}=s$.

For our case, we select the real branch $W_{0}(s)$ of $W(s)$, which is a real function for $s \geq-1 / e$. The series expansion of $W_{0}(s)$ at $s=0$ is

$$
W_{0}(s)=\sum_{n=1}^{\infty}(-n)^{n-1} \frac{s^{n}}{n !}
$$

with the radius of convergence equal to $1 / e[3]$. After some algebraic operations, we obtain the moment generating function $G(s)$ in the form of the series

$$
G(s)=\frac{W_{0}(s)}{s}=\sum_{n=0}^{\infty}(n+1)^{n-1} \frac{(-s)^{n}}{n !}, \quad G(0)=1,
$$

in accordance with 1.3 . The radius of convergence is also equal to $1 / e$. It should be noted that the moment generating function is often taken as $M(s)=G(-s)$, because in this case the generating function has the form of an (at least formal) power series. 


\section{INVERSION OF THE MOMENT GENERATING FUNCTION}

To obtain p.d.f. $f(x)$, we had to invert the Laplace transform (1.1). Three procedures were used: direct, Mellin and Stieltjes.

\subsection{DIRECT PROCEDURE}

Theorem 2.1. The explicit form of the Cayley-Eisenstein-Pólya p.d.f. is:

$$
f(x)=\frac{1}{\pi} \int_{0}^{\pi}\left(y^{2}+(1-y \cot y)^{2}\right) e^{-x y \csc y e^{-y \cot y}} d y, \quad x>0,
$$

Proof. The proof is based on the relation [4]

$$
\frac{W_{0}(s)}{s}=\frac{1}{\pi} \int_{0}^{\pi} \frac{y^{2}+(1-y \cot y)^{2}}{s+y \csc y e^{-y \cot y}} d y, \quad s \in \mathbb{C} \backslash(-\infty,-1 / e),
$$

and on direct application of the Bromwich inversion formula for the Laplace transform and of the Fubini theorem:

$$
\begin{aligned}
f(x)=\frac{1}{2 i \pi} \int_{c-i \infty}^{c+i \infty} & e^{x s} \frac{W_{0}(s)}{s} d s \\
= & \frac{1}{\pi} \int_{0}^{\pi}\left(y^{2}+(1-y \cot y)^{2}\right) \frac{1}{2 i \pi} \int_{c-i \infty}^{c+i \infty} \frac{e^{x s}}{s+y \csc y e^{-y \cot y}} d s d y, \quad c>-1 / e, x>0 .
\end{aligned}
$$

The Bromwich integral in parentheses gives $e^{-x y \csc y e^{-y \cot y}}$, because the inverse Laplace transform of a function $1 /(s+a)$ is $e^{-a x}$, and we have finally Eq. (2.1).

\subsection{MELLin PROCEDURE}

The Mellin procedure is based on the "cannibalistic" feature of the Mellin transform: the Mellin transform of the Laplace transform (and of many others as well) of some function is given by the Mellin transform of that function only [5]. Symbolically:

$$
\operatorname{MT}[\operatorname{LT}[h(t) ; p] ; s]=\Gamma(s) \operatorname{MT}[h(t) ; 1-s], \quad \operatorname{MT}[h(t) ; s]=\operatorname{MT}[\operatorname{LT}[h(t) ; p] ; 1-s] / \Gamma(1-s),
$$

where MT and LT represent the Mellin transform and the Laplace transform, and $\Gamma(s)$ is the Gamma function of argument $s$.

Lemma 2.2. The following Mellin transform pair holds:

$$
s^{s}=\int_{0}^{\infty} x^{s-1} f_{0}(x) d x, \quad s>0, \quad \text { where } \quad f_{0}(x)=\frac{1}{\pi} \int_{0}^{\infty} x^{y} y^{-y} \sin \pi y d y, \quad x>0 .
$$

Proof. In accordance with the Bromwich inversion formula for the Mellin transform, the function $f_{0}(x)$ can be recovered by contour integrals

$$
f_{0}(x)=\frac{1}{2 i \pi} \int_{c-i \infty}^{c+i \infty} x^{-s} s^{s} d s=\frac{1}{2 i \pi} \int_{-\infty}^{\left(0^{+}\right)} x^{-s} s^{s} d s
$$

where the first integral is along the Bromwich contour and the second integral is along the left anticlockwise Hankel contour. The equivalence of these two integrals is based on the Cauchy integral theorem, and on the fact that the integrand has two branch points $s=0, s=-\infty$ and a branch cut in the $s$-plane along the interval $(-\infty, 0)$, and no other singularity. Then

$$
\begin{array}{r}
f_{0}(x)=\frac{1}{2 i \pi} \int_{-\infty}^{\left(0^{+}\right)} x^{-s} s^{s} d s=\frac{1}{2 i \pi} \int_{0}^{\infty} x^{y} e^{-y(\ln y-i \pi)} d y-\frac{1}{2 i \pi} \int_{0}^{\infty} x^{y} e^{-y(\ln y+i \pi)} d y+\frac{1}{2 i \pi} \lim _{r \rightarrow 0} \int_{c_{r}} x^{-s} s^{s} d s \\
=\frac{1}{2 i \pi} \int_{0}^{\infty} x^{y} e^{-y \ln y}\left(e^{i \pi y}-e^{-i \pi y}\right) d y+0=\frac{1}{\pi} \int_{0}^{\infty} x^{y} y^{-y} \sin \pi y d y, \quad x>0, \quad(2.7)
\end{array}
$$

where $c_{r}$ is a small circle with radius $r$ around the origin.

Theorem 2.3. The Cayley-Eisenstein-Pólya p.d.f. $\mathrm{f}(\mathrm{x})$ is represented by the integral

$$
f(x)=\int_{x}^{\infty} \ln \frac{z}{x} \frac{f_{0}(z)}{z} d z, \quad x>0
$$

where function $f_{0}(x)$ is given by Eq. (2.7). 
Proof. We start the proof from the known relationship [3]

$$
\int_{0}^{\infty} u^{s-1} W_{0}(u) d u=(-s)^{-s} \frac{\Gamma(s)}{s}, \quad \Re s \in(-1,0),
$$

from which immediately follows [5, Entry 1.3, p. 11]

$$
\int_{0}^{\infty} u^{s-1} \frac{W_{0}(u)}{u} d u=(1-s)^{1-s} \frac{\Gamma(s-1)}{s-1}, \quad \Re s \in(0,1),
$$

and according to Eq. 2.4 we obtain the Mellin transform of the Cayley-Eisenstein-Pólya p.d.f.:

$$
\int_{0}^{\infty} x^{s-1} f(x) d x=\frac{s^{s}}{s^{2}}, \quad \Re s>0 .
$$

Inversion of the Mellin transform 2.11 can be divided into two steps. The first step is the inversion of $s^{s}$, giving auxiliary function $f_{0}(x)$ according to Lemma 2.1, Eq. (2.7). The second step, according to [5, Entry 1.17, p. 12] combined with [5, Entry 1.3, p. 11], gives the inversion $f_{1}(x)$ of $s^{s} / s$ as

$$
f_{1}(x)=\int_{x}^{\infty} \frac{f_{0}(z)}{z} d z, \quad x>0
$$

and, repeating this procedure once more, we obtain p.d.f. $f(x)$ as

$$
f(x)=\int_{x}^{\infty} \frac{f_{1}(z)}{z} d z, \quad x>0 .
$$

Two consecutive integrations Eq. 2.12 and Eq. 2.13) can be replaced by one integration Eq. 2.8.

\subsection{Stieltues PRocedure}

This procedure is based on the fact that the function $W_{0}(s) / s$ is a Stieltjes function, and can be represented as the Stieltjes transform [4]:

$$
\frac{W_{0}(s)}{s}=\frac{1}{\pi} \int_{1 / e}^{\infty} \frac{\Im W_{0}(-u)}{u} \frac{1}{s+u} d u, \quad s \in \mathbb{C} \backslash(-\infty,-1 / e),
$$

where $W_{0}(-u)=\lim _{\theta \rightarrow 0^{+}} W_{0}(-u+i \theta)$ for $u>-1 / e$ and $\Im(\cdot)$ means the imaginary part of the argument.

Theorem 2.4. Cayley-Eisenstein-Pólya p.d.f. $f(x)$ is represented by the integral

$$
f(x)=\frac{1}{\pi} \int_{1 / e}^{\infty} e^{-x u} \frac{\Im W_{0}(-u)}{u} d u, \quad x>0 .
$$

Proof. The proof is straightforward, because the Stieltjes transform is equivalent to the iterated Laplace transform.

Conversely, by applying the Euler differential operator $-x d / d x$ to p.d.f. $f(x)$ from Eq. 2.1) or Eq. (2.15), respectively, we obtain the function $f_{1}(x)$ :

Theorem 2.5. Function $f_{1}(x)$ is represented by the integrals

$$
\begin{aligned}
f_{1}(x)=-x \frac{d}{d x} f(x)=\frac{1}{\pi} \int_{0}^{\pi} & \left(y^{2}+(1-y \cot y)^{2}\right) x y \csc y e^{-x y \csc y e^{-y \cot y}-y \cot y} d y \\
& =\frac{1}{\pi}\left(-x \frac{d}{d x}\right) \int_{1 / e}^{\infty} e^{-x u} \frac{\Im W_{0}(-u)}{u} d u=\frac{x}{\pi} \int_{1 / e}^{\infty} e^{-x u} \Im W_{0}(-u) d u, \quad x>0
\end{aligned}
$$

Proof. By applying the differential operator $-x d / d x$ to p.d.f. $f(x)$ from Eq. 2.1 and Eq. 2.15 we obtain the function $f_{1}(x)$. In both cases, the conditions for the interchange of the derivation and integration are fulfilled.

By two successive applications of the differential operator $-x d / d x$, i.e. by applying the Euler differential operator $x d / d x+x^{2} d^{2} / d x^{2}$ to p.d.f. $f(x)$ from Eq. 2.1) we obtain the function $f_{0}(x)$ : 
Theorem 2.6. Function $f_{0}(x)$ is represented by the integrals

$$
f_{0}(x)=\left(x \frac{d}{d x}+x^{2} \frac{d^{2}}{d x^{2}}\right) f(x)=\frac{1}{\pi} \int_{0}^{\pi}\left(y^{2}+(1-y \cot y)^{2}\right) M(x, y) d y, \quad x \geq 0,
$$

where

$$
M(x, y)=x y \csc y e^{-x y \csc y e^{-y \cot y}-2 y \cot y}\left(x y \csc y-e^{y \cot y}\right),
$$

and

$$
f_{0}(x)=\frac{1}{\pi}\left(x \frac{d}{d x}+x^{2} \frac{d^{2}}{d x^{2}}\right) \int_{1 / e}^{\infty} e^{-x u} \frac{\Im W_{0}(-u)}{u} d u=\frac{x}{\pi} \int_{1 / e}^{\infty} e^{-x u} \Im W_{0}(-u)(x u-1) d u, \quad x>0 .
$$

Proof. By two successive applications of the differential operator $-x d / d x$, i.e. by applying the Euler differential operator $x d / d x+x^{2} d^{2} / d x^{2}$ to p.d.f. $f(x)$ from Eq. 2.1) and Eq. 2.15, we obtain function $f_{0}(x)$.

After the substitution $x=e^{-\lambda}$, the resulting integral on the right hand side of Eq. 2.7) gives the well-known Landau p.d.f. [2] (more precisely its universal part), which describes the energy loss of a fast charged particle by ionization as it passes through a thin layer of matter:

$$
\phi(\lambda)=\frac{1}{\pi} \int_{0}^{\infty} e^{-\lambda y} y^{-y} \sin \pi y d y, \quad \lambda>-\infty .
$$

From Eq. 2.17) and Eq. 2.20), it follows that the Landau p.d.f. has an alternative integral representation

$$
\phi(\lambda)=\frac{1}{\pi} \int_{0}^{\pi}\left(y^{2}+(1-y \cot y)^{2}\right) M\left(e^{-\lambda}, y\right) d y
$$

Theorem 2.7. The interconnection between the Cayley-Eisenstein-Pólya p.d.f. and the Landau p.d.f. is given by

$$
f(x)=-\int_{-\infty}^{-\ln x}(u+\ln x) \phi(u) d u, \quad x>0 .
$$

Proof. The differential equation in 2.17) is the Euler nonhomogeneous differential equation for the unknown function $f(x)$ and given $f_{0}(x)$ with a particular integral (2.8). After substituting $x=e^{-\lambda}$ to this equation we obtain the following simple differential equation:

$$
\frac{d^{2}}{d \lambda^{2}} \phi_{2}(\lambda)=\frac{d^{2}}{d \lambda^{2}} f\left(e^{-\lambda}\right)=f_{0}\left(e^{-\lambda}\right)=\phi(\lambda), \quad \lambda>-\infty,
$$

where $\phi_{2}(\lambda)=f\left(e^{-\lambda}\right)$. The particular solution of Eq. 2.23 is

$$
\phi_{2}(\lambda)=\int_{-\infty}^{\lambda} \phi(u)(\lambda-u) d u
$$

conformable to Eq. (2.8). After substituting $\lambda=-\ln x$ into Eq. (2.24), we obtain Eq. 2.22).

\section{INTEGRALS AND INTEGRAL TRANSFORMS}

The integral operator $T$ defined by Eq. 2.12): $T\left(f_{0}\right)(x)=\int_{x}^{\infty} \frac{f_{0}(z)}{z} d z, x>0$, is an example of a Mellin multiplier operator with multiplier $1 / s[6]$. This means that the fractional powers of the operator $T$ are [6]:

$$
T^{\alpha}\left(f_{0}\right)(x)=\frac{1}{\Gamma(\alpha)} \int_{x}^{\infty} \ln ^{\alpha-1} \frac{z}{x} \frac{f_{0}(z)}{z} d z, \quad x>0, \alpha>0, T^{0}=I,
$$

where $I$ is the identity operator, and that it holds for the Mellin transform of Eq. 3.1):

$$
\operatorname{MT}\left[T^{\alpha}\left(f_{0}\right)(x) ; s\right]=s^{-\alpha} \operatorname{MT}\left[f_{0}(x) ; s\right] .
$$

We thus have a one-parameter family of the functions $f_{\alpha}(x), 0 \leq \alpha \leq 2, f_{2}(x) \equiv f(x)$, given by

$$
f_{\alpha}(x)=T^{\alpha}\left(f_{0}\right)(x), \quad x>0 .
$$

Moreover, the integral operator $T^{\alpha}\left(f_{0}\right)(x)$ in Eq. 3.1$)$ is the Hadamard right-sided fractional integral of the order $\alpha$ of the function $f_{0}(x)$ [7]. By analogy, there exists a one-parameter family of the functions $\phi_{\alpha}(x)$, $0 \leq \alpha \leq 2, \phi_{0}(x) \equiv \phi(x)$, given by

$$
\phi_{\alpha}(x)=U^{\alpha}\left(\phi_{0}\right)(x), \quad x>-\infty
$$


where

$$
U^{\alpha}\left(\phi_{0}\right)(x)=\frac{1}{\Gamma(\alpha)} \int_{-\infty}^{x}(x-z)^{\alpha-1} \phi_{0}(z) d z, \quad x>-\infty, \alpha>0, U^{0}=I,
$$

is the Liouville left-sided fractional integral [7] of the order $\alpha$ of the Landau p.d.f. Equation (2.24) is a special case for $\alpha=2$.

Because $W_{0}(s) / s$ is a Laplace transform of the p.d.f. $f(x)$, it is natural to ask what the Laplace transform of the functions $f_{0}(x)$ and $f_{1}(x)$ is.

Theorem 3.1. We have

$$
\begin{gathered}
\int_{0}^{\infty} e^{-s x} f_{1}(x) d x=\frac{d}{d s} W_{0}(s)=\frac{W_{0}(s)}{s\left(1+W_{0}(s)\right)}=\frac{e^{-W_{0}(s)}}{1+W_{0}(s)}, \quad \Re s>-1 / e, \\
\int_{0}^{\infty} e^{-s x} f_{0}(x) d x=-\frac{d}{d s} W_{0}(s)+\frac{d^{2}}{d s^{2}}\left(s W_{0}(s)\right)=\frac{W_{0}(s)}{s\left(1+W_{0}(s)\right)^{3}}, \quad \Re s>-1 / e .
\end{gathered}
$$

Proof. With the aid of the standard rules for the Laplace transform, e.g. [8, Entry 1.24, p.6], applied to Eq. (2.16) and Eq. 2.17), respectively, we obtain the results.

Corollary 3.2. The following Mellin transform pairs hold:

$$
\begin{gathered}
\int_{0}^{\infty} u^{s-1} \frac{W_{0}(u)}{u\left(1+W_{0}(u)\right)} d u=\Gamma(s)(1-s)^{-s}, \quad \Re s \in(0,1), \\
\int_{0}^{\infty} u^{s-1} \frac{W_{0}(u)}{u\left(1+W_{0}(u)\right)^{3}} d u=\Gamma(s)(1-s)^{1-s}, \quad \Re s \in(0,1) .
\end{gathered}
$$

Proof. Because Eq. (3.6) holds and $\mathrm{MT}\left[f_{1}(x) ; s\right]=s^{s} / s$, we apply the relation 2.4 and obtain Eq. (3.8). An analogous procedure with Eq. (3.7), and the fact that $\mathrm{MT}\left[f_{0}(x) ; s\right]=s^{s}$ gives Eq. (3.9).

Corollary 3.3. The following Laplace transform pairs hold:

$$
\begin{gathered}
f_{1}(x)=\frac{1}{\pi} \int_{1 / e}^{\infty} e^{-x u} \Im\left(\frac{W_{0}(-u)}{u\left(1+W_{0}(-u)\right)}\right) d u, \quad x \geq 0, \\
f_{0}(x)=\frac{x}{\pi} \int_{1 / e}^{\infty} e^{-x u} \Im\left(\frac{W_{0}(-u)}{1+W_{0}(-u)}\right) d u, \quad x>0 .
\end{gathered}
$$

Proof. Function $\frac{W_{0}(s)}{s\left(1+W_{0}(s)\right)}$ is a Stieltjes function [4]. From the definition of the Stieltjes transform and from Eq. (3.6), it follows that

$$
\int_{0}^{\infty} e^{-s z} f_{1}(x) d x=\frac{1}{\pi} \int_{1 / e}^{\infty} \Im\left(\frac{W_{0}(-u)}{u\left(1+W_{0}(-u)\right)}\right) \frac{1}{s+u} d u, \quad \Re s>-1 / e,
$$

and after inversion of the Laplace transform we obtain Eq. (3.10). Equation 3.11 is a consequence of the relation $f_{0}(x)=-x d / d x f_{1}(x)$.. The integral in Eq. (3.10) also converges for $x=0$.

Theorem 3.4. We have

$$
\int_{0}^{\infty} e^{-s x} \frac{f_{0}(x)}{x} d x=\frac{1}{1+W_{0}(s)}, \quad \Re s>-1 / e .
$$

Proof. Because

$$
\frac{d}{d s} \int_{0}^{\infty} e^{-s x} \frac{f_{0}(x)}{x} d x=-\int_{0}^{\infty} e^{-s x} f_{0}(x) d x=-\frac{W_{0}(s)}{s\left(1+W_{0}(s)\right)^{3}}, \quad \Re s>-1 / e
$$

and

$$
\frac{d}{d s} \frac{1}{1+W_{0}(s)}=-\frac{W_{0}(s)}{s\left(1+W_{0}(s)\right)^{3}}
$$

we obtain Eq. (3.13). 
Corollary 3.5. We have

$$
\begin{gathered}
\frac{1}{\pi} \int_{0}^{\infty} s^{-y} y^{-y} \sin \pi y \Gamma(1+y) d y=\frac{W_{0}(s)}{\left(1+W_{0}(s)\right)^{3}}, \quad s \in \mathbb{C} \backslash D_{1 / e} \\
\frac{1}{\pi} \int_{0}^{\infty} s^{-y} y^{-y} \sin \pi y \Gamma(y) d y=\frac{1}{1+W_{0}(s)}, \quad s \in \mathbb{C} \backslash D_{1 / e},
\end{gathered}
$$

where $D_{1 / e}$ is an open disc of complex numbers with absolute value $r<1 / e$.

Proof. Substitution of $f_{0}(x)$ from Eq. (2.7) to Eq. 3.7) and Eq. (3.13) gives Eq. (3.16) and Eq. (3.17), respectively.

After substituting $s=e^{p}$ into Eq. (3.16) and Eq. 3.17) we obtain the Laplace transform pairs

$$
\begin{gathered}
\int_{0}^{\infty} e^{-p y} y^{-y} \sin \pi y \Gamma(1+y) d y=\frac{\pi W_{0}\left(e^{p}\right)}{\left(1+W_{0}\left(e^{p}\right)\right)^{3}}, \quad \Re p>-1,-\pi<\Im p \leq \pi, \\
\int_{0}^{\infty} e^{-p y} y^{-y} \sin \pi y \Gamma(y) d y=\frac{\pi}{1+W_{0}\left(e^{p}\right)}, \quad \Re p>-1,-\pi<\Im p \leq \pi .
\end{gathered}
$$

The fact that the abscissa of convergence of the two previous Laplace integrals is equal to -1 can be verified by the Stirling formula. It should be emphasized, however, that the right hand sides of Eq. (3.18) and Eq. (3.19) are not holomorphic functions in the half-plane $\Re p \geq a>-1, a$ otherwise arbitrary. This means that there exist functions holomorphic in the half-plane $\Re p>-1$, defined by the integrals on the left hand sides of Equations (3.18) and (3.19), and equivalent to the functions on the right hand sides in the region defined in Eq. (3.18) and Eq. (3.19). Regarding Eq. (3.18) or Eq. (3.19) as inverse problem needs to apply the real methods for the Laplace transform inversion.

Theorem 3.6. We have

$$
\frac{\Gamma(s)}{\pi} \int_{0}^{\infty} y^{-s} y^{-y} \sin \pi y \Gamma(y) d y=\int_{0}^{\infty} \frac{y^{s-1}}{1+W_{0}\left(e^{y}\right)} d y, \quad 0<\Re s<1 .
$$

Proof. Application of the Mellin transform to both sides of Eq. (3.19), and taking Eq. (2.4) into account, gives the result.

Note that both integrals in Eq. 3.20 are Mellin transforms.

Theorem 3.7. We have

$$
\frac{\Gamma(s)}{\pi} \int_{1 / e}^{\infty} u^{-s-1} \Im W_{0}(-u) d u=\frac{s^{s}}{s^{2}}, \quad \Re s>0 .
$$

Proof. From the Mellin transform of Eq. 2.15, taking Eq. 2.11) and Eq. 2.4 into account, it follows that

$$
\frac{s^{s}}{s^{2}}=\operatorname{MT}\left[\frac{1}{\pi} \int_{1 / e}^{\infty} e^{-x u} \frac{\Im W_{0}(-u)}{u} d u ; s\right]=\frac{\Gamma(s)}{\pi} \int_{1 / e}^{\infty} u^{-s} \frac{\Im W_{0}(-u)}{u} d u, \quad \Re s>0 .
$$

Theorem 3.8. We have

$$
f_{1}(x)=1-\frac{1}{\pi} \int_{0}^{\infty} x^{y} y^{-y-1} \sin \pi y d y, \quad x \geq 0
$$

Proof. From Eq. (3.13) it follows that

$$
\int_{0}^{\infty} \frac{f_{1}(x)}{x} d x=\frac{1}{1+W_{0}(0)}=1
$$

Eq. 2.12 thus can be rewritten in the form

$$
f_{1}(x)=1-\int_{0}^{x} \frac{f_{0}(z)}{z} d z=1-\frac{1}{\pi} \int_{0}^{\infty} y^{-y} \sin \pi y \int_{0}^{x} z^{y-1} d z d y,
$$

where we substituted $f_{0}(z)$ from Eq. (2.7). Because $\int_{0}^{x} z^{y-1} d z=x^{y} / y, y>0$, we obtain Eq. (3.23). 
Corollary 3.9. We have

$$
f_{1}(x)=1-\int_{-\ln x}^{\infty} \phi(u) d u=\int_{-\infty}^{-\ln x} \phi(u) d u, \quad x \geq 0,
$$

where $\phi(u)$ is Landau p.d.f. 2.20).

Proof. Equation 2.12 can also be rewritten in the form

$$
f_{1}(x)=1-\int_{0}^{x} \frac{f_{0}(z)}{z} d z=1-\int_{-\ln x}^{\infty} \phi(u) d u,
$$

where the substitution $z=e^{-u}$ is used. The integral on the right of Eq. 3.26 is a direct consequence of the same substitution into Eq. (2.12).

Corollary 3.10. We have

$$
f(x)=-\ln x f_{1}(x)-\int_{-\infty}^{-\ln x} u \phi(u) d u, \quad x>0 .
$$

Proof. Equation (3.28) is a consequence of Eq. 2.22) and of Eq. (3.26).

Theorem 3.11. We have

$$
f_{1}(x)=\int_{0}^{x} f(x-u) \frac{f_{0}(u)}{u} d u, \quad x>0 .
$$

Proof. $W_{0}(s) / s$ is a Laplace transform of the p.d.f. $f(x)$. From Equations (3.6) and (3.13), it immediately follows that function $f_{1}(x)$ is a Laplace convolution of the functions $f(x)$ and $f_{0}(x) / x$.

Theorem 3.12. We have

$$
\int_{0}^{x}(1+f(x-u)) \frac{f_{0}(u)}{u} d u=1, \quad x>0 .
$$

Proof. According to Eq. 3.25) and Eq. 3.29), we find that

$$
\int_{0}^{x}(1+f(x-u)) \frac{f_{0}(u)}{u} d u=\int_{0}^{x} \frac{f_{0}(u)}{u} d u+\int_{0}^{x} f(x-u) \frac{f_{0}(u)}{u} d u=\left(1-f_{1}(x)\right)+f_{1}(x)=1 .
$$

\section{Conclusion}

Defining the moment generating function of the probability distributions by means of the Laplace or LaplaceStieltjes transform proposed in [1] makes it possible to obtain the interconnection between the Cayley-EisensteinPólya probability distribution, which is native in queueing theory with the Landau distribution originating in atomic physics. This interrelation is given by an integral relation/equation of the first kind Eq. (2.22) and in principle by a Euler differential relation/equation in Eq. (2.17), differential relation Eq. (2.23) and by the Laplace convolution Eq. 3.30).

From the formal point of view, the absolute convergence of the Laplace transform integral and moreover its uniform convergence generally constitute an advantage in the calculations and in the process of inference of the formulae. As regards the integral transform pairs that are a byproduct of this study, the author cannot guarantee their novelty. However, but he has not found them elsewhere.

\section{ACKNOWLEDGEMENTS}

Many thanks to both referees for their valuable comments and suggestions.

\section{REFERENCES}

[1]Abate J., Whitt W.: An Operational Calculus for Probability Distributions via Laplace Transforms, Adv. Appl. Probab. 28, (1), 1996, p. 75-113.

[2]Landau L.D.: On the Energy Loss of Fast Particles by Ionisation, J.Phys. U.S.S.R. 8, 1944, also in Editor: D. ter Haar.: Collected Papers of L.D.Landau, Oxford: Pergamon Press, 1965, p.417-424.

[3]Corless R. M. et al: On the Lambert W Function, Adv. Comput. Math. 5, 1996, p.329-359.

[4]Kalugin G.A. et al: Bernstein, Pick, Poisson and Related Integral Expressions for Lambert W, to apear in Integral Transforms Spec. Funct.

[5] Oberhettinger F.: Tables of Mellin Transforms, New York: Springer-Verlag, 1974.

[6]Rooney P.G.: A survey of Mellin multipliers, In: "Fractional calculus" (Editors: A. C. McBride, G.F.Roach). London: Pitman publishing, 1985, p. 176-187.

[7]Kilbas A.A. et al: Theory and Applications of Fractional Differential Equations, Amsterdam: Elsevier, 2006.

[8]Oberhettinger F., Badii L.: Tables of Laplace Transforms, New York: Springer-Verlag, 1973. 